IMPACT OF LANIFIBRANOR, A PAN-PPAR AGONIST, ON THE PULMONARY VASCULAR TONE

Chandran Nagaraj ${ }^{1}$, Neha Sharma ${ }^{2}$, Bence Nagy ${ }^{1}$, Jean-Louis Junien ${ }^{3}$, Pierre Broqua ${ }^{3}$, Irena Konstantinova ${ }^{3}$, Andrea Olschewski ${ }^{1,4}$, Grazyna Kwapiszewska ${ }^{1,4}$, Horst Olschewski ${ }^{1,2}$. ${ }^{1}$ Ludwig Boltzmann Institute for Lung Vascular Research, Graz, Austria; ${ }^{2}$ Medical University of Graz, Department of Pulmonology, Graz, Austria; ${ }^{3}$ Inventiva, Daix, France; ${ }^{4}$ Otto Loewi Research Center, Medical University of Graz, Graz, Austria

Background: Pulmonary hypertension $(\mathrm{PH})$ is common in patients with fibrosing interstitial lung diseases (ILDs) such as systemic sclerosis (scleroderma) and has a significant impact on survival. The pan-peroxisome proliferator-activated receptor (PPAR) agonist lanifibranor is currently being tested in a phase II clinical trial in scleroderma patients. Although, the antifibrotic effects of lanifibranor have been previously shown in preclinical mouse models of scleroderma and related $\mathrm{PH}(1)$, its effects in modulating the pulmonary vascular tone are largely unknown.

Objectives: To evaluate the acute effect of lanifibranor (pan-PPAR agonist) and selective PPAR agonists on the pulmonary vascular tone.

Methods: Freshly isolated intra-pulmonary arteries from explanted human and rat lungs were used in wire myography experiments to assess the acute effect on pulmonary vascular tone of pan-PPAR agonist -lanifibranor and the selective PPAR agonists PPAR $\alpha$-fenofibrate, PPAR $\beta / \delta$ GW50156 and PPAR $\gamma$-troglitazone $(0.01 \mu \mathrm{M}-30 \mu \mathrm{M})$ versus vehicle (DMSO). The intra-pulmonary arteries were pre-constricted with $300 \mathrm{nM}$ of the thromboxane A2 receptor agonist U46619. The contribution of endothelium and nitric oxide was investigated using both native and endothelium-denuded vessels, with and without the nitric oxide synthase inhibitor L-NAME.

Results: Isometric tension measurements in rat pulmonary arteries showed that lanifibranor and the selective PPAR agonists PPAR $\beta / \delta$-GW50156 and PPAR $\gamma$-troglitazone, induced a significant relaxation compared to their vehicle controls, whereas the PPAR $\alpha$ agonist fenofibrate did not show any vasoactive effect on the pre-constricted arteries. Lanifibranor showed a significant vasodilatory effect already at $1 \mu \mathrm{M}$ whereas GW50156 and troglitazone showed significant vasodilatory effects starting from $10 \mu \mathrm{M}$ as compared to their vehicle controls. At the maximum concentration used $(30 \mu \mathrm{M})$, lanifibranor caused a relaxation response of $-85 \%$ whereas GW50156 and troglitazone caused just $-45 \%$ and $-50 \%$ vasorelaxation $(n=6)$, respectively. The vasodilation induced by pan-PPAR and selective PPAR agonists remained unaffected by pre-treatment of L-NAME or denuded endothelium. Lanifibranor had vasodilatory effects that were strongly superior to any of the selective PPAR agonists in the isolated pulmonary artery from the explanted human lungs $(n=3)$.

Conclusion: The current study revealed that the pan-PPAR agonist lanifibranor caused a rapid dose-dependent relaxation in both rat and human intra pulmonary arteries which were superior to any other PPAR agonists suggesting additive effects of the pan-PPAR agonism. The observed vasoactive effects of pan-PPAR are independent of nitric oxide and endothelium, delineating the specific effect of PPAR agonism on the pulmonary artery smooth muscle cells. These results suggest that lanifibranor could be a promising therapy for vasculopathies such as $\mathrm{PH}$ observed in scleroderma patients.

REFERENCE:

[1] Avouac $\mathrm{J}$ et al. Pan-PPAR agonist IVA337 is effective in experimental lung fibrosis and pulmonary hypertension. Ann Rheum Dis. 2017 Nov;76 (11):1931-1940.

Disclosure of Interests: Chandran Nagaraj: None declared, Neha Sharma: None declared, Bence Nagy: None declared, Jean-Louis Junien Shareholder of: shareholder at inventiva, Employee of: employee of inventiva, Pierre Broqua Shareholder of: shareholder at inventiva, Employee of: employee at Inventiva, Irena KONSTANTINOVA Shareholder of: shareholder at inventiva, Employee of: employee at Inventiva, Andrea Olschewski: None declared, Grazyna Kwapiszewska Grant/research support from: research grant from Inventiva, Horst Olschewski Grant/research support from: financial grant from Bayer,Actelion,Boehringer,roche,inventiva, Consultant for: Bellerophon, MSD, TEVA, menarini,Chiesi,Novartis,GSK,BAYER, Roche,Boehringer

DOI: 10.1136/annrheumdis-2019-eular.4003

\section{THU0341 MICRORNA27A3P REGULATES WNT SIGNALLING BY TARGETING SFRP-1 IN SYSTEMIC SCLEROSIS}

\section{Steven O'Reilly. Northumbria University, Newcastle, United Kingdom}

Background: Systemic sclerosis (SSc) is an autoimmune idiopathic con nective tissue disease that results in fibrosis of the skin and lungs. Wnt signaling is a highly conserved signaling pathways that is involved in organogenesis and tissue homeostasis. In recent years it has emerged that aberrant Wnt signaling is key in SSc and animal models of the disease. The Wnt pathway comprises of secreted glycoproteins that bind to specific receptors that initiate the pathway and result in beta catenin stabilization. However, secreted antagonists are secreted to block and negatively regulate Wnt signaling. We and others have found reduced sFRP-1 in SSc, a key Wnt antagonist. Although lower in SSc skin and isolated fibroblast from patients the mechanism governing this is unknown. This work seeks to identify epigenetic mechanisms.

Objectives: Determine the epigenetic regulation of SFRP-1 in SSc.

Methods: Serum sFRP-1 was measured in healthy and diffuse SSc patients to ascertain the levels of SFRP-1. Mir27a was identified as a possible microRNA that targets sFRP1 using in silico modeling. miR27a was transfected into healthy dermal fibroblasts using transfection methods and SFRP-1 was measured. Collagen was also measured by western blotting after transfection. MiR27a was also measured in SSc dermal fibroblasts using specific Taqman probes and normalized to RNU44 miR Cloning of the 3'UTR of sFRP1 into a luciferase reporter vector was performed and then transfected into HEK293 cells with miR27a mimics or with concentration matched scramble and after 24 hours luciferase was monitored.

Results: Significantly reduced expression of sFRP-1 was found in SSc serum compared to controls $(n=10)$. SSc dermal fibroblasts had 3 fold higher levels of miR27a compared to healthy fibroblasts. Increased miR27a in healthy dermal fibroblasts resulted in significantly less sFRP-1 secreted into conditioned media and this was co-incident with an increase of collagen1 levels. Finally cloning of the 3'UTR binding site in a luciferase plasmid and overexpression of miR27a led to reduced levels of luciferase in HEK293 cells in vitro.

Conclusion: Reduced SFRP-1 in systemic sclerosis is mediated by miR27a and this leads to fibrosis via enhanced Wnt signaling. Targeting the epigenetic changes that mediate this may be a therapy in SSc.

Disclosure of Interests: None declared

DOI: 10.1136/annrheumdis-2019-eular.7619

\section{THU0342 SYSTEMIC SCLEROSIS IS A DISEASE OF A PREMATURELY SENESCENT, INFLAMMATORY AND ACTIVATED IMMUNOME}

Bhairav Paleja ${ }^{1}$, Andrea Low ${ }^{2}$, Pavanish Kumar ${ }^{1}$, Suzan Saidin ${ }^{1}$, Ahmad Lajam ${ }^{1}$ Sharifah Nur Hazirah ${ }^{1}$, Camillus Chua ${ }^{1}$, Liyun Lai ${ }^{1}$, Salvatore Albani ${ }^{1}$.

${ }^{1}$ Translational Immunology Institute, Singhealth/Duke-NUS Academic Medical Centre, Singapore, Singapore; ${ }^{2}$ Singapore General Hospital, Singapore, Singapore

Background: Systemic sclerosis (SSc) is an autoimmune disease characterised by excessive fibrosis of skin and internal organs, and vascular dysfunction (1). Association of $T$ and $B$ cell subsets have been reported in SSc, however there is lack of systematic studies of functional relations between immune cell subsets in this disease $(2,3,4)$. This lack of mechanistic knowledge hampers targeted intervention.

Objectives: In the current study we ought to determine differential immune cell composition and their interactions in peripheral blood of SSc patients and its impact on disease severity and progression.

Methods: Mononuclear cells from blood of SSc patients $(n=20)$ and healthy controls $(n=10)$ were analysed by mass cytometry using a 36 marker (cell-surface and intracellular) panel to aid in identification of major PBMC lineages including $T$ cells, B cells, monocytes and NK cells and their subsets. Transcriptome analysis (m-RNA sequencing) was performed on sorted $T$ and $B$ cell subsets. Unsupervised clustering of mass cytometry data was performed using in-house developed analysis software MARVIS. This software combines dimension reduction and clustering steps to identify all possible cellular subsets. Further, custom R scripts helped in identifying nodes that were differentially expressed between the study groups and also phenotype of these nodes.

Results: Unsupervised clustering analysis revealed significant differences in the frequencies of $\mathrm{T}$ and $\mathrm{B}$ cell subsets in patients. Correlation network analysis highlighted an overall dysregulated immune architecture coupled with domination of inflammatory senescent $T$ cell modules in SSc patients. Transcriptome analysis of sorted immune cells revealed an activated phenotype of CD4 and MAIT cells in patients, accompanied with increased expression of inhibitory molecules, reminiscent of 